\title{
Calendar of Events
}

We provide this selected list of upcoming events as a service to our readers. It gives brief information on various conferences and meetings, corresponding to the scope and interests of this journal, and which are of more than local significance or highly specialized orientation. As a consequence of the always increasing number of such events, space limitations will compel us to list only those which are scheduled to take place within one year of the publication date of a given issue of $I S U$-except that the first issue of each year will publish an extended listing. Workshops, courses and training sessions are, furthermore, expressly excluded.

Of course we can assume no responsibility for the complete accuracy of the information on which individual entries are based. Supplementary entries and updated details are incorporated with each issue, insofar as these come in the meantime to our attention.

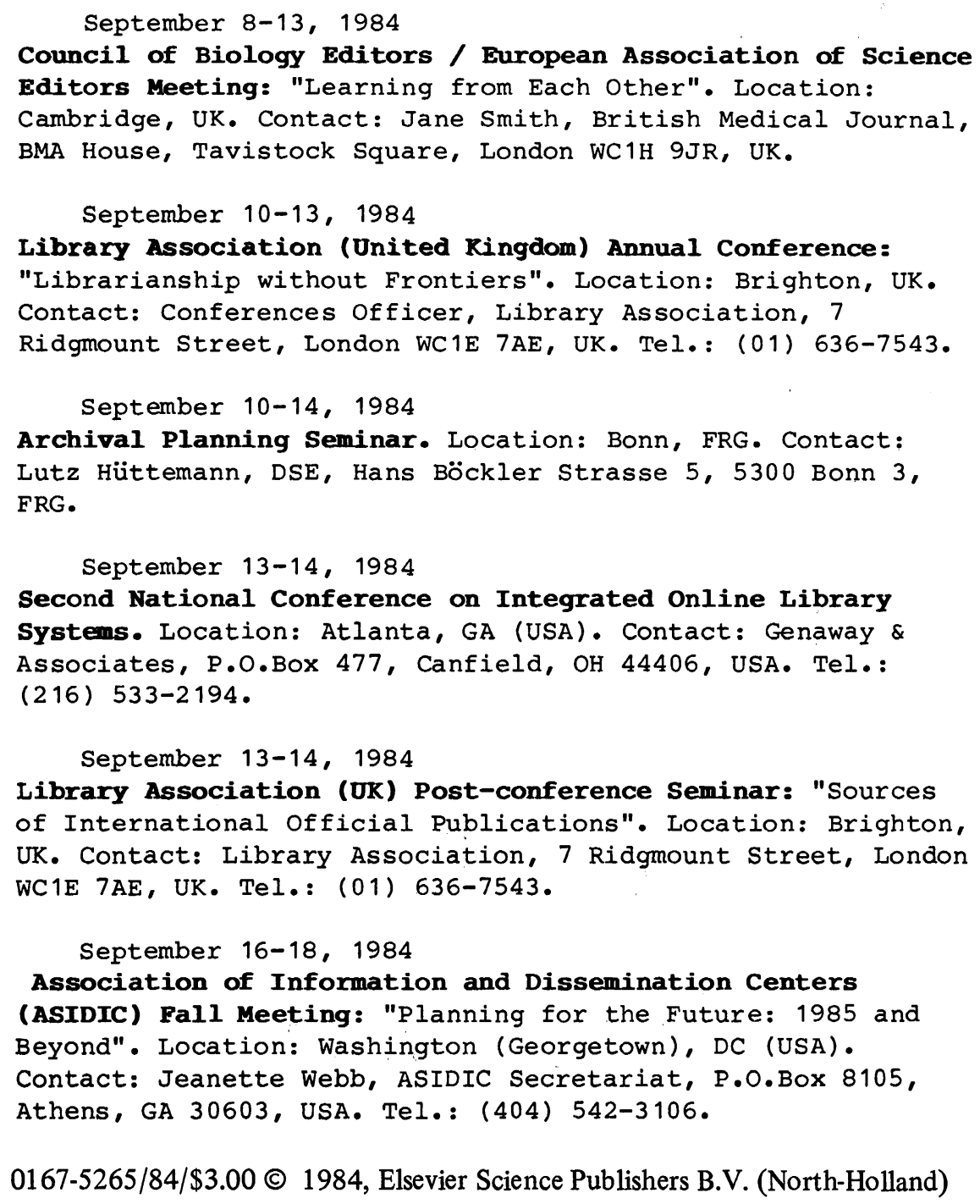


September 17-21, 1984

Tenth International Congress on Archives: "The Challenge to Archives: Growing Responsibilities and Limited Resources". Location: Bonn, FRG. Contact: Prof. Dr. H. Booms, Bundesarchiv, Postfach 320, Am wöllershof 12, 5400 Koblenz 1, FRG.

September 17-21, 1984

3e Congrès-Exposition International sur les Banques de Données - Infodial/Videotex '84. Location: Paris, France. Contact: Infodial/Videotex, 4 place de Valois, 75001 Paris, France. Tel.: (1) 261-5242. Telex: $212597 \mathrm{f}$.

September $18-21,1984$

ASIIB 57th Annual Conference: "Information Management Potential and Practice". Location: Norwich, UK. Contact: Conference Organiser, ASLIB, 3 Belgrave Square, London SW1X 8PL, UK. Tel.: (01) 235-5050. Telex: 23667.

September 20-21, 1984

Planning the Electronic Library. Location: Boston, MA (USA). Contact: Aaron Cohen Associates, RFD 1, Box 636, Teatown Road, Croton-on-Hudson, NY 10520, USA. Tel.: (914) 271-8170.

September 21-23, 1984

Annual Conference of the International Institute of Commanications. Location: Berlin, FRG. Contact: IIC, Tavistock House East, Tavistock Square, London WC1H 9LG, UK.

September 24-27, 1984

42nd Congress of the Fédération Internationale de Documentation (FID): "The Use of Information in a Changing World". Location: The Hague, The Netherlands. Contact: FID, P.0.Box 90402, 2509 LK The Hague, The Netherlands. Tel.: (070) 140671 .

September 24-27, 1984

7th Essen Symposium: "Local Library Systems". Location: Essen, FRG. Contact: Universitätsbibliothek Essen, Postfach 101454, Universitätsstrasse 9, 4300 Essen 1, FRG. Tel.: (0201) 18337 00. Telex: 857239 ueghb d.

September 25-28, 1984

CAMP ' 84 - Computer Graphics Applications for Management and Productivity. Location: Berlin, FRG. Contact: AMK Berlin, Ausstellungs-Messe-Kongress-GmbH, Postfach 191740 , Messedamm 22, 1000 Berlin 19, FRG. Tel.: (030) 30381. Telex: 182890 amkc d.

September 25-30, 1984

IFIP/Network ' 84 (Office Automation). Location: Sofia, Bulgaria. Contact: P.O.Box 373, Sofia 1090, Bulgaria. 
October, 1984

International Symposium on Harmonization of Training

Programs in Information Science, Librarianship and Archival

Studies. Location: Paris, France. Contact: UNESCO-PGI, 7

place de Fontenoy, 75700 Paris, France.

October $3-5,1984$

Automation of Information Services. Location: Sofia, Bulgaria. Contact: CISTI, 52a G.A. Nasser Boulevard, 1040

Sofia, Bulgaria. Telex: 22404.

October $9-12,1984$

Deutscher Dokumentartag. Location: Darmstadt, FRG. Contact: Deutsche Gesellschaft für Dokumentation, Westendstrasse 19, 6000 Frankfurt a.M. 1, FRG. Tel.: (0611) 747761.

October 9-12, 1984

Computer Applications Software systems Conference (COMPAS '84). Location: Berlin, FRG. Contact: AMK Berlin, Dept. K 12, Messedamm 22, 1000 Berlin 19, FRG.

October 12-16, 1984

Vidcom 184. Location: Cannes, France. Contact: Vidcom, 179 avenue Victor-Hugo, 75116 Paris, France.

October $15-16,1984$

Library Software Conference. Location: Philadelphia, PA

(USA). Contact: Meckler Publishing, 520 Riverside Avenue, Westport, CT 06880, USA. Tel.: (203) 226-6967.

October $16-18,1984$

European Association of Information Services (EUSIDIC)

Annual Conference: "Information as an Exploitable

Resource". Location: Baden, Austria. Contact: Helen

Henderson, EUSIDIC Administrative Secretary, P.0.BOx 429, London W4 1UJ, UK, tel. (01) 546-7968, telex 825962 ; or, Peter Popper, IIASA, 2361 Laxenburg, Austria, tel. (02236) 71521, ext. 283, telex 079137 iiasa a.

October 19-21, 1984

Networks India ' 84 - International Symposium on Data Communication and Computer Networks. Location: Madras, India. Contact: Dr. H.N. Mahabala, Department of Computer Science and Engineering, Indian Institute of Technology, Madras-600 036, India. Tel.: 414719, 415342. Telex: $041-7362$.

October 23-26, 1984

47th American Society for Information Science (ASIS) Annual Meeting: "Challenges to an Information Society". Location: Philadelphia, PA (USA). Contact: ASIS, 1010 Sixteenth Street NW, Washington, DC 20036, USA. Tel.: (202) 659-3644.

October $24-25,1984$

Association of Research Libraries (ARL), USA, Membership Heeting. Location: Washington, DC (USA). Contact: ARL, 1527 New Hampshire Avenue NW, Washington, DC 20036, USA. Tel.: (202) 232-2466. 
October 29-31, 1984

Online '84: "The Next Horizon". Location: San Francisco, CA (USA). Contact: Online inc., 11 Tannery Lane, Weston, CT 06883, USA.

October 30 - November 2, 1984

7 th International Conference on Computer Communications ICCC 184. Location: Sydney, Australia. Contact: Peter Davidson, ICCC '84, GPO Box 2.367, Sydney, NSW 1001, Australia; or, John D. MCKendree, ICCC, Box 9745, Washington, DC 20016, USA.

November $4-8,1984$

The 2nd International Congress on Advances in Non-impact Printing Technologies. Location: Arlington, VA (USA). Contact: Mr. Werner E. Haas, Webster Research Center, Xerox Corporation, 800 Phillips Road, W-114, Webster, NY 14580, USA. Tel.: (716) 422-4203.

November $5-9,1984$

Compsac 84. Location: Chicago, IL (USA). Contact: IEEE, Institute of Electrical and Electronic Engineers, 345 East 47 th Street, New York, NY 10017, USA.

November $11-14,1984$

Information Industry Association (IIA), USA, 16th Annual Conference and Exhibition: "The Information Nation: New Actors, New Factors". Location: San Francisco, CA (USA). Contact: IIA, 316 Pennsylvania Avenue SE, Suite 400, washington, DC 20003, USA. Tel: (202) 544-1969.

November 13-14, 1984 Electronic Publishing Conference. Location: London, UK. Contact: Jane Holland, Conference Coordinator, Brintex ltd. 178-202 Great Portland Street, London W1N 6NH, UK.

November $13-14,1984$

Electronic Publishing and Printing Exhibition 1984. Location: London, UK. Contact: John Northover, Brintex ltd., 178-202 Great Portland Street, London W1N 6NH, UK.

November $13-14,1984$

Premier Colloque National sur 1'Information en Chimie.

Location: Paris, France. Contact: Société de chimie industrielle, 28 rue Saint-Dominique, 75007 Paris, France.

November $14-15,1984$

Text Retrieval ' 84 (sponsored by the Institute of Information Scientists, southern branch). Location: London, UK. Contact: Catherine Hamilton, 25 Queens Avenue, London N10 3PE, UK.

November $15-16,1984$

American Society of Access Professionals (ASAP)

International Symposium: "1984 - Reassessing Information Policies and Orwell's Warnings". Location: Washington, DC (USA). Contact: Russ Roberts, ASAP, P.0.BOX 1252, Arlington, VA 22210 , USA. 
November $20-21,1984$

1 as Jornadas Españolas de Documentación Automatizada.

Location: Madrid, Spain. Contact: Instituto de Información y Documentación en Ciencia y Tecnología, CSIC C/ Joaquín Costa no. 22, Madrid-6, Spain. Tel.: 2614808. Telex: 22628 cidmd.

November $20-21,1984$

Translating and the Computer 6: Translation and

Communication. Location: London, UK. Contact: Conference Organiser, ASLIB, 3 Belgrave Square, London SW1X 8PL, UK. Tel.: (01) 235-5050. Telex: 23667.

November 21-23, 1984

Videotex Europe. Location: Amsterdam, The Netherlands. Contact: Online Conferences ltd., Pinner Green House, Ash Hill Drive, Pinner, Middlesex HA5 2AE, UK. Tel.: (01) 868-4466. Telex: 923498 online $g$.

November $23-25,1984$

Third Annual Acquisitions Librarians Conference. Location: Basingstoke, United Kingdom. Contact: Jane Kellock or Nigel Oxbrow, Alan Armstrong \& Associates, 72 Park Road, London NW1 4SH, UK. Tel.: (01) 258-3740.

November $26-28,1984$ Library Association of Australia, Education for Librarianship Section Conference: "The Information Professional". Location: Melbourne, Australia. Contact: Janette Wright, Department of Librarianship, Royal Melbourne Institute of Technology, GPO Box 2476V, Melbourne, Victoria 3001, Australia.

December 4-6, 1984

8th International Online Information Meeting. Location: London, UK. Contact: Learned Information, Besselsleigh Road, Abingdon, Oxford OX13 6LG, UK. Tel.: (0865) 730275. Telex: 837704 inform $g$.

\section{December 5-7, 1984}

OECD Berlin Conference: "1984 and After: The Societal Challenge of Information Technologies". Location: Berlin, FRG. Contact: Secretariat, Organization for Economic Cooperation and Development, Information, Computex and Communications Policy Committee, 2 rue André Pascal, 75775 Paris, France.

December $13-14,1984$ Planning the Electronic Library. Location: New York, NY (USA). Contact: Aaron Cohen Associates, RFD 1, Box 636, Teatown Road, Croton-on-Hudson, NY 10520, USA. Tel.: (914) 271-8170. 
January 5-10, 1985

American Library Association (ALA) Mid-winter Con-

ference. Location: Washington, DC (USA). Contact: ALA, 50

East Huron Street, Chicago, IL 60611, USA. Tel.: (312)

944-6780.

January 30 - February 1, 1985

Special Libraries Association (SLA) winter meeting.

Location: Philadelphia, PA (USA). Contact: Conference and Exhibits Coordinator, SLA, 235 Park Avenue South, New York, NY 10003, USA. Tel.: (212) 477-9250.

Fèruary 4-6, 1985

1985 Office Automation Conference: "Today's Partnership: People and Technology". Location: Atlanta, GA (USA). Contact: Dr. Sidney E. Harris, Decision Sciences Laboratory, Georgia State University, University Plaza, Atlanta, GA 30303, USA. Tel.: (404) 658-4000.

February 18, 1985

1985 Congress for Librarians: "Downloading/Uploading Online Databases and.Catalogs". Location: Jamaica, NY (USA). Contact: James Benson or Bella Weinberg, Division of Library \& Information Science, St. John's University, Jamaica, NY 11439, USA. Tel.: (212) 990-6161, ext. 6200 .

March, 1985

Library History Seminar VII: "Books, Libraries and Culture". Location: Chapel Hill, NC (USA). Contact: Dr. Donald G. Davis, Jr., Graduate School of Library and Information Science, University of Texas at Austin, Box 7576, University Station, Austin, TX 78712, USA.

March 4-5, 1985

IFIP TC 6 International Conference on Data Communications in the ISDN Era. Location: Tel-Aviv, Israel. Contact: Ortra ltd., 431 Namir Sq., P.O.Box 3473, Tel-Aviv, Israel. Tel.: (03) 289128. Telex: 361142 .

March 25-28, 1985

United Kingdom Serials Group (UKSG) Annual Conference. Location: Nottingham, UK. Contact: Rodney M. Burton, Secretary, UKSG, Science Reference Library, 25 Southampton Buildings, Chancery Lane, London WC2A 1AW. Tel.: (01) $405-8721$, ext. 3055 .

March 28-30, 1985 Making Library and Information Research Applicable. Location: Bath, UK. Contact: Ms. S. Corrall, British Library Research \& Development Department, 2 Sheraton Street, London W1V 4BH, UK. 
April, 1985

Second Infoterm Symposium / First International Workshop on Terminology Documentation: "Networking in Terminology". Location: Vienna, Austria. Contact: Infoterm, Postfach 130, Leopoldsgasse 4, 1020 Vienna 2, Austria. Tel.: (0222) 24833117 .

April 15-19, 1985

IATUL Conference: "Cooperation Amongst Technological

Libraries - the International Scene". Location: Oxford, UK. Contact: International Association of Technological University Libraries, c/o Chalmers Tekniska Hфgskolas Bibliotek, Fack S-420, 20 Göteborg, Sweden.

April 16-18, 1985

Informatics 8: "Advanced Computational Techniques for Information Retrieval". Location: Oxford, UK. Contact: ASLIB, 3 Belgrave Square, Iondon SW1X 8PL, UK. Tel.: (01) 235-5050. Telex: 23667.

April 30 - May 2, 1985

Sixth National Online Meeting. Location: New York, NY (USA). Contact: Thomas H. Hogan, Learned Information inc., 143 Old Marlton Pike, Medford, NJ 08055, USA. Tel.: (609) 654-6266.

May $16-17,1985$

Association of Research Libraries (ARL), USA, Membership Meeting. Location: Cincinnati, OH (USA). Contact: ARL, 1527 New Hampshire Avenue NW, Washington, DC 20036, USA. Tel.: (202) 232-2466.

June 2-6, 1985

Seventh Congress of the International Association of Agricultural Librarians and Documentalists: "Information Delivery: the Weak Links in the Chain". Location: Ottawa, Ontario, Canada. Contact: Canadian Planning Committee, IAALD '85, Room 249, Sir John Carling Building, Agriculture Canada, Ottawa, Ontario K1A 0C5, Canada.

June $8-13,1985$

Special Libraries Association (SLA) 76th Annual

Conference: "The Information Specialist: a Bridge to the New Communications". Location: Winnipeg, Manitoba, Canada. Contact: Conference and Exhibits Coordinator, SLA, 235 Park Avenue South, New York, NY 10003, USA. Tel.: (212) $477-9250$.

June $12-14,1985$

IDT '85: 6ème Congrès National sur l'Information, la Documentation et le Transfert des Connaissances: "La Société de l'Information". Location: Versailles, France. Contact: Comité IDT '85, Association nationale de la recherche technique, 101 avenue Raymond Poincaré, 75116 Paris, France. Tel.: (1) 501-7227. 
June $23-26,1985$

ICC ' 85 - International Conference on Communications.

Location: Chicago, IL (USA). Contact: ICC-85, P.0.Box 1147,

Lombard, IL 60148, USA.

July 6-11, 1985

American Library Association (ALA) Annual Conference.

Location: Chicago, IL (USA). Contact: ALA, 50 East Huron Street, Chicago, IL 60611, USA. Tel.: (312) 944-6780.

July 29 - August 2, 1985

WCCE ' 85 - World Conference on Computers in Education.

Location: Norfolk, VA (USA). Contact: Dr. G. Engel,

Chairman, WCCE ' 85 Organizing Committee, Department of

Computer Science, Christopher Newport College, Newport News, VA 23606, USA.

August 18-24, 1985

International Federation of Library Associations and Institutions (IFLA) Council and General Conference:

"Libraries and the Universal Availability of Information". Location: Chicago, IL (USA). Contact: IFLA, P.O.BOx 95312, $2509 \mathrm{CH}$ The Hague, The Netherlands. Tel.: (070) 140884 .

August 20-22, 1985

New Zealand Library Association Conference: "Libraries: For All People?". Location: Dunedin, New Zealand. Contact: Norah Familton, Box 5542, Moray Place, Dunedin, New Zealand.

August 25-27, 1985

International Conference on Technical Communication -

Intecom Forum '85. Location: Helsingor, Denmark. Contact: Thomas O'Connor, Intecom Forum '85, Dantekom, P.0.Box 146, 3600 Fredrikssund, Denmark. 